\title{
A Technique For Lock-In Prediction On A Fluid Structure Interaction Of Naca 0012 Foil With High Re
}

\author{
Nu Rhahida Arini' ${ }^{1}$, Stephen R. Turnock², Mingyi Tan ${ }^{3}$ \\ ${ }_{1}^{1}$ Politeknik Elektronika Negeri Surabaya, Indonesia. \\ 2,3University of Southampton, England \\ E-mail: 1arini@pens.ac.id, 2S.R.Turnock@soton.ac.uk, ${ }^{3}$ M.Tan@soton.ac.uk
}

Received September 30, 2020; Revised November 4, 2020; Accepted December 8, 2020

\begin{abstract}
A numerical lock-in prediction technique of a NACA 0012 hydrofoil, immersed in a flow having a $R e$ of $3.07 \times 10^{6}$ is proposed in this paper. The technique observes the foil's response as part of a fluid-structure interaction analysis. The response is modelled by foil's vibration which is represented by spring and damper components. The technique identifies and predicts the foil's lock-in when it vibrates. The prediction is examined using the Phase Averaged Method which employs the Hilbert Transform Method. The aim of this paper is to propose a numerical way to identify a lock-in condition experienced by a NACA 0012 foil in a high Reynolds number flow. The foil's mechanical properties are selected and its motions are restricted in two modes which are in the pitch and heave directions. The rotational and transverse lock-in modes are identified in the model. The existence of lock-in is verified using pressure distribution plot, the history of trailing edge displacement and fluid regime captures. The history of total force coefficients are also shown to clarify the result. From the result, it can be shown that the technique can predict reliably the lock-in condition on the foil's interaction. Three main fluid induced vibration frequencies are generated in the interaction. None of them are similar to the foil's natural frequency and the phenomenon of lock-in is apparently not found in the typical operational condition.
\end{abstract}

Keywords: Tidal Turbine, FSI, CFD.

\section{INTRODUCTION}

The interaction of a foil and fluid comes in many engineering areas. The fluid structure interaction (FSI) analysis is important in following areas: the higher safety in aerospace technology, the increasing demand for renewable energy supply, improved ship efficiency in marine industry as well as other examples. Although the fluid and foil interaction in various applications have been of interest in the last decade but the lock-in condition is not clearly understood and not well reported. The technique of lock-in identification in actual practice is not obvious and detailed. This makes predicting the lock-in on a foil difficult. This paper reviews a 
procedure to solve the problem. The aim of this paper is to propose a technique or procedure for a FSI CFD model to predict the lock-in response. The FSI model utilizes a NACA 0012 foil which has $0.75 \mathrm{~m}$ chord length. The foil works in a fluid flows at high Re which corresponds to $3.07 \times 10^{6}$. The k$\omega S S T$ turbulence model is used in all models which are simulated in the OpenFOAM.

Locked-in condition is a phenomenon when frequency of a foil lays near the foil's natural frequency. Lock-in phenomenon that is distinguished by high frequency vibrations is strongly related to vortex-induced vibration (VIV). Although in some cases high amplitude may appear in lock-in as reported by Mittal et al. (2016). The interaction between a foil and fluid generates a pressure distribution on the foil's surfaces. The surface pressure during the interaction creates forces and drives the foil to oscillate. This oscillation appears as vibrations on the foil. The vibration induces vortices in the fluid regime which, in some instances, generates lock-in condition. The foil's vibration also affects vortices to shed with similar frequency to the foil's natural frequency when lock-in occurs. The lock-in has been of interest in the last decade and was studied by Bishop (1964), and Besem et al. (2016).

\section{Related works}

In this paper, the interaction is represented by vibration motions. Therefore, in this section of related works, the model of foil's vortex-induced vibration for representing a FSI from previous works done by other researchers are explained. However, the bending and other structure effects caused by vibrations are not discussed. Nonetheless, by understanding the behaviour of the fluid regime near the structure, problems of vibration can beavoidable.

The vibration causes instability to the structure and occurs together with other problems such as lifetime reduction and fatigue failure. Vibrations as a fluid structure interaction response has been studied by Rana et al. (2011), Ducoin et al. (2009; 2013), Akcabay et al. (2014a, 2014b), and Bessem (2016). All studies developed response models by a vibrational system which consisted of spring and damper components. Spring and damper components were used by Besem et al. (2016) to numerically and experimentaly study the vortex on an oscillating foil. They introduced a two springs system in the model to represent the foil's response which was allowed to oscillate by a highest angle of $5^{\circ}$. They varied the angle of attack in respect to incoming fluid to $50^{\circ}$. They found that the amplitude of lock-in frequency was higher than the unlocked frequency amplitude by four times.

A vibrational model using a system with spring and damper components has also been proposed by Akcabay et al. (2014a; 2014b). Their model represented a response of flexible hydrofoil in heave and pitch direction. The simulation result had good agreements with Ducoin's model (Ducoin et al., 2009; 2013). Ducoin $(2009,2013)$ also proposed a vibration model which utilized spring damper components to evaluate response of a hydroil. His foil's response is mainly caused by fluid cavity. Rana et al. (2011) 
also evaluated a response of NACA 0012 using a vibrational approach with vertical spring and damper components which were attached at a quarter of chord length. One component was generated for imitating pitch response (torsional vibration) and another spring and damper system to model heave response (transverse vibration). They used their model to investigate 2D flutter characteristic. The results were in the form of pressure distribution and heave and pitch motion history. The moment which was produced by the total force was constant and was not affected by angles of attack acted on the foil.

Fluid structure instruction problem can also be solved using dynamic mesh. Solving FSI problems by the use of OpenFOAM is recommended since the physical solvers for determining the structural response and the fluid behaviour equation are coupled in its library. The recommended solver is straightforward within an executable simulation. The solver is also supplied by a handy feature for mesh refinement including snappyHexMesh. The feature is very helpful for modelling a dynamic mesh. The high-quality mesh is processed within three stages to optimize number of cells built in the domain grid. The grid obtains a precision result and numerical stability with having little computational effort and less time consuming.

In this paper the foil interaction is considered to appear in pitch and heave motion. The torsional and transverse vibration response is modelled by two spring and damper systems which is virtually placed at the foil chord length with a quarter length distance from leading edge (LE) as illustrated in Figure 1a.

The spiral spring and damper component perform pitch motion which drives torsional vibration on the foil. The vertical spring damper system introduces transverse vibration. The 2D CFD domain used in this paper is adapted from Arini et.al (2018). The generation and validation of the domain mesh resolution was explained in their paper. The developed model employs a spring damper component as in Figure 1a and generated in OpenFOAM. The CFD domain is shown in Figure $1 \mathrm{~b}$. The mesh around the foil's surface is refined using snappyHexMesh utility and the trailing edge refinement is depicted in Figure 1c.

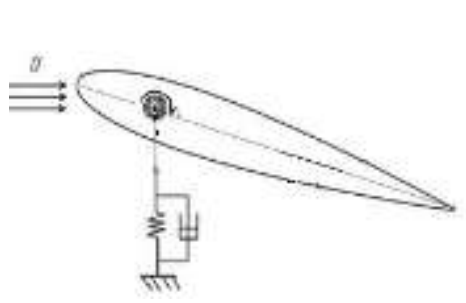

a.

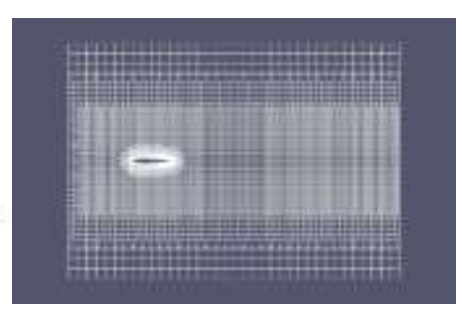

b.

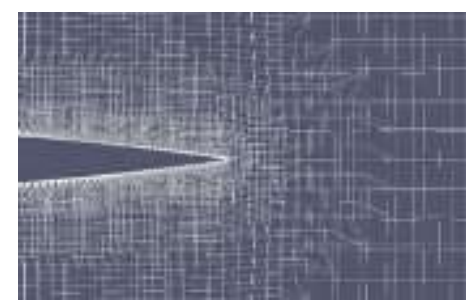

c.

Figure 1. a. A vibration system with spring and damper components to model a foil response, $b$. The foil response model domain in 2D, c. refinement on the foil trailing edge 


\section{Originality}

The originality of this paper is to propose an approach for lock-in prediction for a NACA 0012 foil model using FSI method. The foil has $0.75 \mathrm{~m}$ chord length and immersed in a fluid flow with Re of $3.07 \times 10^{6}$. The model is executed in OpenFOAM using sixDoFRigidBody boundary condition to model vibration for FSI response. To handle the foil motion, pointDisplacement dictionary is utilized and run in pimpleDyMFoam solver. Another assumption is taken which is constant incoming flow.

The prediction of lock-in for FSI response of a foil is examined using the Fourier Transform Method and the Phase Averaged Method. Hussain and Reynolds (1970; 1972) firstly introduce the Phase Averaged Method. The method is used for extracting a fluctuating signal of a coherent turbulent structure, such as vortex shedding frequency. The fluctuating signal is extracted from an original signal. The fluctuating component is obtained by subtracting the original signal from a periodic reference signal. Any original signal is naturally composed of a periodic component and a fluctuating component of the coherent signal which is represented by a phase averaged signal. Therefore, the fluctuating component of any coherent signal can be determined by subtracting a periodic component from the original signal.

The Phase Averaged Method was employed by researchers including Jung and Park (2005). They conducted an experiment to find vortex shedding frequency of an oscillating foil in a incoming flow with constant velocity. They measured the fluid velocity at $0.1 \mathrm{c}$ behind trailing edge using PIV. This work adapts the probe distance as suggested by Jung. Ostermann et al. (2014) implemented the Phase Averaged Method to filtered fluctuating components from an oscillating flow in their experiment. They determined a phase averaged signal using Hilbert Transform Method and convert fluctuating signal to vortex shedding frequency using the PSD method. They also suggested that the periodic signal as a reference should be a signal with an undisturbed periodic character (pure) and not distracted by any fluctuating components.

Bourgeois et al. (2013a; 2013b) also used the Phase Averaged Method to predict vortex shedding frequency in his model. Perrin et al. $(2006 ; 2007)$ conducted an experiment and analyzed the vortex shedding frequency using the Phase Averaged Method. Bourgeois and Perin found that the Phase Average Method gave fairly precise results for obtaining vortex shedding frequency. In the paper, the Phase Averaged Method is implemented to extract frequency of a vortex shedding. The original signal is the form of velocity plot obtained at a node which is located $0.1 \mathrm{~m}$ away from TE as suggested by Jung and Park (2005). The nodal velocity is strongly characterized by the vortex shedding behaviour. The reference signal is taken from a point close to LE which exhibits an undisturbed periodic vibration motion as confirmed by Ostermann et al. (2014). 
The reference signal is transformed to a phase averaged signal employing the Hilbert Transform Method. Further the phase averaged signal is subtracted from the original signal to extract the time domain vortex shedding velocity. To convert the time domain vortex shedding velocity to frequency domain data, the PSD method is used. The methods explained earlier are employed in this work for estimating the lock-in as result of NACA 0012 foil interaction with fluid. The routine to proceed FSI on NACA 0012 is depicted in Figure 3. The analysis and usage the procedure of lock-in prediction approach is detailed in Section 5. In that section, the procedure is applied in the prediction of lock-in in fluid structure interaction between a hydrofoil and sea water which is employed in the model.

\section{System Design}

Discussion of the system design is separated into two parts which are the discussion of system natural frequency and spring-damper components.

\subsection{Foil's Natural Frequency}

A foil's natural frequency is a mechanical property when the foil is undamped and initially displaced. The second order differential undamped free vibration equation only has mass and stiffness component remain at one side and another side is zero. The calculation returns second order harmonic differential equations. The equations is solved by finding a matrix of the natural frequency as an eigenvalue. The matrix equation of free vibration shown in Equation (1)

$$
\left[\begin{array}{cc}
m & 0 \\
0 & l
\end{array}\right] \frac{d^{2}}{d_{L}^{2}}\left[\begin{array}{l}
y \\
\theta
\end{array}\right]+\left[\begin{array}{cc}
k_{\mathrm{t}} & 0 \\
0 & k_{f}
\end{array}\right]\left[\begin{array}{l}
y \\
\theta
\end{array}\right]=\left[\begin{array}{l}
0 \\
0
\end{array}\right]
$$

$\mathrm{K}_{\mathrm{t}}, \mathrm{K}_{\mathrm{r}}, \mathrm{m}$ and $\mathrm{I}$ are transverse material stiffness, torsional material stiffness, mass of the foil, and foil moment of inertia respectively. $\mathrm{K}_{\mathrm{t}}$ and $\mathrm{K}_{\mathrm{r}}$ are taken from the material properties data.

Equation (1) can be simplified and become:

$$
M \frac{d^{2} x}{d_{L}^{2}}+K_{i} x=0
$$

Generalized form is in the form of $\mathrm{X}$ direction. $\mathrm{M}$ and $\mathrm{K}_{\mathrm{i}}$ is the generalized form of mass and the foil's stiffness constant. Equation (2) is considered to be a harmonic system and has a solution form of Xsinwnt where $\mathrm{X}$ is the displacement amplitude. By inserting the solution into Equation (2), the vibration equation becomes

$$
\omega_{\mathrm{n}}^{2} M_{\mathrm{i}} X=K_{\mathrm{i}} X
$$

Generelized eigenvector (ui) and eigenvalue ( $\lambda \mathrm{i}$ ) can be utilized to solve the problem by substituting the terms in the Equation of vibration (equation 3 ). The Equation of vibration becomes $\lambda \mathrm{Mu}=\mathrm{Ku}$. The foil natural frequency $\left(\omega_{\mathrm{n}}\right)$ solution satisfies $\omega_{i}=\sqrt{\lambda_{i}}$. In vibration with one degree of freedom involved on a system, the torsional or the transverse natural frequency can be solved separetly.

Davies et al. (2013) experimentally studied a hydrofoil in high Re. In the experiment, he used polymer composite for material of the foil. which has. The result showed that static flexural stiffness of the material was roughly 
$1000 \mathrm{~N} / \mathrm{m}$. The material stiffness of the torsional vibration was calculated to be $200 \mathrm{Nm} /$ radians. In this paper the foil is assumed to be fabricated from polymer composite which gives advantage from the ability to absorb vibrational energy.

When evaluating structural natural frequency of a structure immersed in liquid such as water, one should consider a term called added mass. The fluid gains an excess weight to the structure as it moves in the water. De La Torre et.al (2013) investigated a hydrofoil's mass to be examined from the added mass of the system. The result showed three modes of added mass constant due to the foil vibration orientation. The orientation modes are similar to the torsion vibration, second bending, and first bending. In this paper, the vibration is assumed to have the second bending and torsion mode. Da Lozzo et al. (2012) and Ghassemi and Yari (2011) developed a model for their object to study added mass. Ghassemi and Yari (2011) considered added mass and included it in his turbine propeller model. Their result showed that a factor of 1.07 should be implemented when a wet condition is being designed as situated in this research. This factor is taken and inserted into the mass term in this vibration equation. Using the added mass method, the transverse (heave) and torsional natural frequency are found to be $0.9425 \mathrm{~Hz}$ and $1.4758 \mathrm{~Hz}$ respectively.

\subsection{Spring and Damping Components}

The foil response model in the FSI analysis is developed by a vibration motion using spring and damper components as shown in Figure 1a. The response constraint of motion is in two degrees of freedom. They are heave and pitch. The two spring damper systems are attached to the foil's dynamic centre (point of rotation) which is located at a quarter of chord length from LE. The two spring and damper systems represent heave and pitch responses which are modelled by torsional and transverse vibration. The transverse and torsional vibration working on the blade are assumed the first mode of force vibration and analyzed at quasi steady. The stiffness and damping coefficients are determined based on the blade material which is assumed to be fabricated from a material of polymer composite.

The heave transverse stiffness coefficient is approximated from an experiment conducted by Davies (stated in his paper, Davies et.al, 2013, in Figure 7, Polymer line). The experiment tested a hydrofoil which was fabricated from a polyurethane casting as proposed by Klaassen (2015). The casting material is strengthened by using glass fibre reinforcement (chopped strand mat or quasi-unidirectional) to improve polymer stiffness properties. Klaassen (2015) suggested a polyurethane coating for a composite material which can avoid a foil from cavitation and pressure changing during operation.

In this research, the foil is expected to be fabricated from forty percent of epoxy and sixty percent e-glass by volume $25.29 \%$ resin and $74.71 \%$ fibre by weight). The density of the material is using a volume fraction of sixty percent e-glass fibre and forty percent epoxy resin. The fibre and resin have 
density of $2.56 \mathrm{~g} / \mathrm{cm}^{3}$ and $1.3 \mathrm{~g} / \mathrm{cm}^{3}$ respectively. The typical material has $2.06 \mathrm{~g} / \mathrm{cm}^{3}$ density. The e-glass is added in large amount to strengthen the foil. Further the foil is covered with a polyurethane substance to protect the foil surface from damage caused by changing pressure and cavitation.

The torsional stiffness coefficient is obtained from transverse stiffness coefficient of the material taking an assumption that the material is isotropic. The mechanical properties of an isotropic material are independent from the body's orientation. Therefore, the mechanical properties are identical in all directions. However, the stiffness coefficient is affected by the moment of inertia and the body mass. The stiffness coefficient in a direction can be estimated from the transverse stiffness coefficient using the mass to moment inertia ratio. The mass of the foil is calculated using the density of material and volume of the foil. The mass and moment inertia in respect to pitch motion are calculated and found to be $16.73 \mathrm{~kg}$ and $3.75 \mathrm{kgm}^{2}$ respectively. From the isotropic approach using the mass to moment of inertia ratio, the torsional stiffness is found to be $224.15 \mathrm{Nm} / \mathrm{rad}$ and is rounded to 200 $\mathrm{Nm} / \mathrm{rad}$ in this paper. The torsional stiffness coefficient estimated from the isotropic material approach is confirmed with the torsional stiffness coefficient estimated from a method developed by Tabassian (2013).

Tabassian (2013) observed torsional vibration working on shafts using Adomian Decomposition Method (ADM). The torsional vibration stiffness coefficient from Tabbasian's method is estimated from the shaft natural frequency with a circle cross section area and turbine blade material shear modulus. He also suggested a torsional evaluation to calculate a shaft natural frequency with different supports. One type of Tabbassian's models is having mass shaft concentration with springs support at both ends.

Shear modulus value is obtained from Chung et.al paper (2001). In the paper, they observed vibration and damping of several composites and polymers. They evaluated the materials (shown in Table 1 of their paper) using three parameters. The parameters are loss modulus, $\tan \delta$, and storage modulus. The parameters are utilized in Dynamic Mechanical Analysis (DMA) to represent the stress response under a fluctuated load working on a viscoelastic material. Tan $\delta$ is a coefficient for measuring the capacity of damping. A viscoelastic material deforms in the range of an elastic material and a viscous fluid. Under working force, a viscous fluid experiences deformation according to Newtonian behaviour. In Newtonian behaviour theory, stress is proportional to the shear-strain rate. This contrasts with elastic material which proportionally deforms to strain.

The storage modulus works as a restrined force within the molecules of the material. The force is used by the material to return to its initial condition. Therefore, the storage modulus is an elastic solid like behaviour. The loss modulus is produced if the force acting on the material is higher and break the molecules bond of the material. The material is said of being deformed and have a viscous-like behaviour. Increasing the ability of the vibration damping, the material should have high shear modulus and/or 
high $\tan \delta$. These parameters affect the loss modulus. The shear modulus is obtained from the loss modulus of epoxy listed in Table 1 in Chung's paper. The loss modulus of epoxy is estimated to be $0.11 \mathrm{GPa}$.

The natural frequency is solved using this method and the calculated torsional stiffness is found to be $278.42 \mathrm{Nm} / \mathrm{rad}$ which is close to the torsional stiffness constant calculated using the approach of isotropic. The small discrepancy of torsional stiffness constant between the ADM method and isotropic approach is likely because in the ADM method the observed shaft has a circular cross-sectional area.

Damping characteristic of hybrid polymer matrix composites is studied experimentally by Kumar (2015). From his results, it was found that natural frequency is insfluenced proportionally to a damping ratio (Table III in Kumar's paper). In this paper, the damping ratio is 0.0074 with a critical damping value is $258.69 \mathrm{Ns} / \mathrm{m}$ in heave direction.

Further, to obtain damping coefficient, a parameter named damping ratio is evaluated. Damping ratio affects the damped condition of a system under vibration. Damping ratio is mathematically defined as ratio of damping value to critical damping of a system. The limit value is laid at 1 . When a system has damping ration larger than 1 , the system is said to be overdamped and restrain to steady condition (no vibration). On the other hands the system is in under damped condition if the damping ration is less that 1 . In this case, the system exhibits vibrations which exponentially decays prior to reaching a steady condition.

Damping coefficient in heave in this paper is calculated and is obtained to be $1.91 \mathrm{Ns} / \mathrm{m}$. The value is rounded to $2 \mathrm{Ns} / \mathrm{m}$. The torsional damping constant is also determined using the method for defining heave damping consant. The torsional critical damping is obtained from Kumar (2015) and rounded to be $50 \mathrm{Ns} / \mathrm{m}$. The constant is calculated and rounded to become $0.2 \underline{\mathrm{Ns}} / \mathrm{m}$.

\section{Analysis}

The routine, which is detailed in this section, explains step by step process accessing the simulation result to predict lock-in on the foil interaction. The process is detailed as follows:

1. Pick two nodes on the mesh for velocity history recording.

The two nodes are located at the front (near LE) and at the back $(0.08 \mathrm{~m}$ behind TE) as seen in Figure 2a (the nodes are marked with pink spot). The $\mathrm{LE}$ and TE points are recorded to collect the reference and original velocity signals respectively.

2. Record the velocity history from the nodes (in point 1 )

The designated nodes are at LE and at $0.08 \mathrm{~m}$ away from TE which represent the reference and original signals respectively. The velocity history of reference and original signals are seen in Figure $2 \mathrm{~b}$. The figure shows that in the first three seconds, the simulatoin experiences a transient condition which is demonstrated by unsteady velocity. After three seconds, the velocity 
hystory of the original signal (red line) stabilizes and have more regular fluctuations. This indicates the occurrence of steady state after 3 seconds. The transient condition at the first three seconds is removed in the Phase Averaged signal. Singals of reference, original and the phase averaged signals can be seen in Figure 2c.

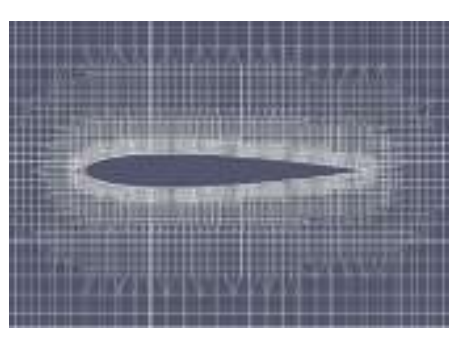

a

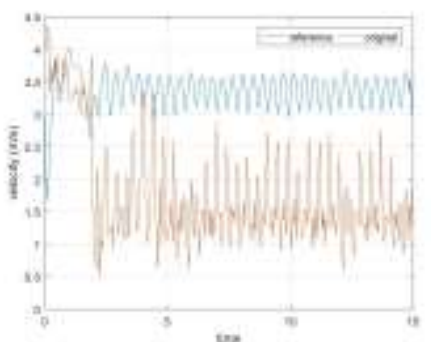

b.

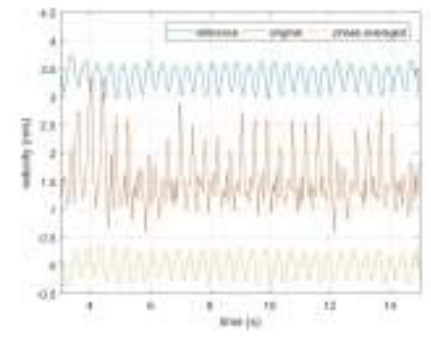

c.

Figure 2. a. Two points selected for velocity recording. b. Velocity recording of two selected points. c. the cut-off velocity time history of the selected points, omitting the transient part at the first three seconds.

3. The process of phase averaging to gain vortex shedding velocity

The Phase Averaged Method is employed by substracting phase averaged signal from the original signal to access the velocity of vortex shedding as depicted in Figure 3a. The vortex shedding velocity is the signal experienced by a point at $0.08 \mathrm{~m}$ away from trailing edge. This phased averaged signal is basically the fluctuating component which has been separated from the periodic input signal.

4. The time domain vortex shedding signal transformation

The time domain vortex shedding signal shown in Figure $3 \mathrm{a}$ is transformed to frequency domain signal utilizing the Power Spectral Density Method. Although the time domain foil's velocity reflects vibrations, the data cannot confirm the ocuurance of foil's lock-in. The velocity should be converted and filtered using the PSD method to obtain the frequency domain signal. The vortex shedding frequency after it is filtered by the PSD Method is shown in Figure 6b. The frequency data indicates the dominant frequency of foil's vibration.

In Figure $3 b$, the foil heave $\left(f_{h}\right)$ and torsional $\left(f_{\theta}\right)$ natural frequencies, as explained in Section 4, are also depicted as vertical lines. The value which are obtained from natural frequency vibration, are $0.9425 \mathrm{~Hz}$ and is $1.4758 \mathrm{~Hz}$. Comparing both natural frequencies to the main frequencies (dominant frequencies) in the frequency domain plot will identify the lock-in occurrence. From Figure $3 \mathrm{~b}$, it can be seen that the heave and torsional natural frequencies form lock-in band. The lock-in appears if one of the dominant frequencies lie near or on the frequency lock-in band (Bishop and Hasan (1964) and Bessem (2016)). 


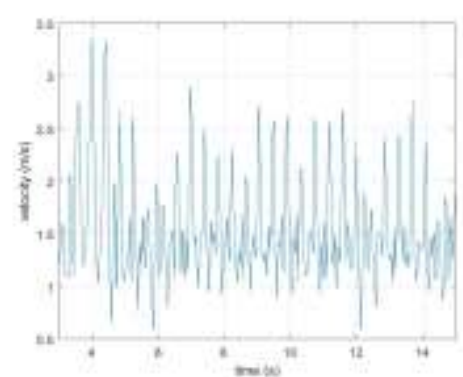

a.

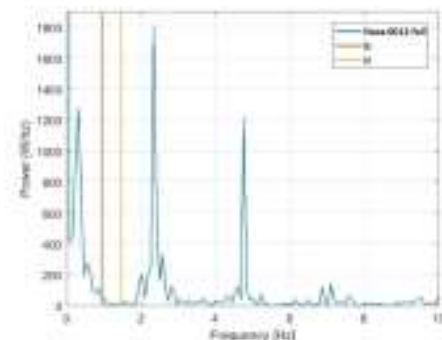

b.

Figure 3. a. Phase averaged velocity signal obtained from the Phase Averaged Method, b. Vortex shedding in a frequency domain

5. Examine lock-in from dominant vortex shedding frequencies and natural frequencies.

Lock-in condition on the foil is examined by comparing dominant frequencies and heave and torsional natural frequencies. Figure $3 \mathrm{~b}$ shows that torsional natural frequency is higher than heave natural frequency. This fact indicates that the pitch foil's response (represented by torsional frequency) is predominant over the heave foil's response. Figure 3b shows three predominant frequencies (peaks) occurs in the foil. These frequencies are $4.76 \mathrm{~Hz}, 2.34 \mathrm{~Hz}$, and $0.31 \mathrm{~Hz}$. All frequencies occur away from torsional and heave natural frequencies, therefore the vibration (the foil's response) does not exhibit lock-in condition and a resonance does not appear in the foil. A resonance is indicated by strong vibrations which is detected from plot of the foil's displacement. The foil's displacement model (vibration) which is forced by the fluid loading are depicted in Figure 5b.

6. Verification the response, whether it is lock-in or not.

The verification intends to confirm whether the foil experience lock-in condition or not. There are three features that can be used for a foil response verification. The features are the vortex shedding contour in the wake behind the trailing edge, pressure distribution on both foil's surfaces and the foil's displacement (vibration).

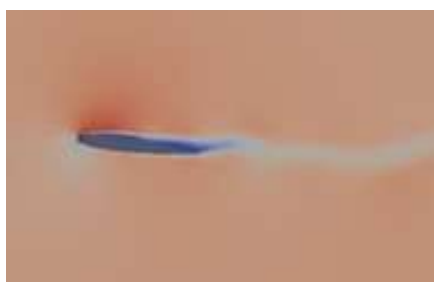

a.

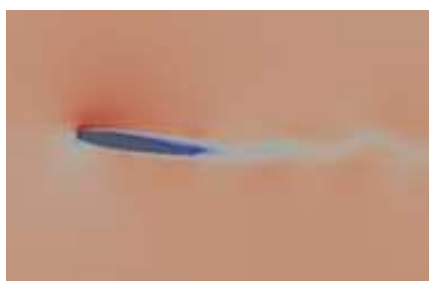

b.

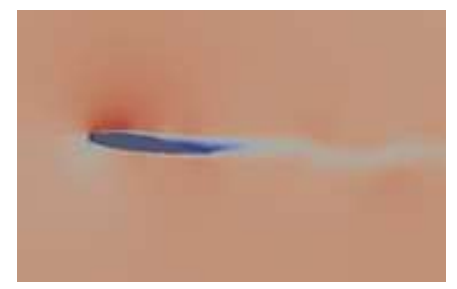

c.

Figure 4. Fluid behaviour over a vibration cycle, at t/T: a. 0.1, b. 0.6, c. 1 
- Vortex shedding profile

Vortex shedding is vizualized on the regime over the upper surface and behind the trailing edge. The generation of the vortex can be shown by one cycle (T) of foil's oscillation (vibration) which is depicted in Figure 4.

Figure $4 \mathrm{a}$ shows the foil at $\mathrm{t} / \mathrm{T}$ equals to 0.1 when it is at the lowest position of oscillation. At this position, a small vortex is generated at the leading edge (LEV-leading edge vortex) and flows forward to the trailing edge. The vortex leads to lower pressure at the upper surface and brings the foil to move upward reaching the maximum elevation at $\mathrm{t} / \mathrm{T}=0.6$ as shown in Figure $4 \mathrm{~b}$. At this incident, the attached vortex in the wake generate maximum lift force over one cycle of vibration. At the end of this stage, the vortex detaches and flows ar near wake behing trailing edge and makes the upper surface regime smooth and more regular. The upper surface pressure increases (overall pressure difference at foil's surface decrease) lead the foil to go downward and reaches the minimum position. This situation is illustrated in Figure 4c. The pressure distribution over foil's surface strongly influences lift force thus the vibration/movement (displacement). The lift force can be seen in Figure $5 c$ and will be detailed later.

LEV which is constantly generated can be detected by a pressure peak from pressure distribution profile over foil's surface as seen in Figure 5a. As explained, the vortex produces oscillation on the foil which can be indicated from trailing edge displacement. The displacement is illustrated in Figure $5 b$ and will be discussed later.

- Pressure distribution.

Figure 5a shows pressure distribution on foil's upper and lower surface during the interaction. The pressure distribution profile agrees with Blevins' result (1990) that oscillates on the location where the vortex is shed. Figure 5 a shows the pressure distribution profile at $\mathrm{t} / \mathrm{T}=0.1,0.6$ and 1 . The $\mathrm{t} / \mathrm{T}$ shown corresponds to Figure $4 \mathrm{a}, 4 \mathrm{~b}$, and $4 \mathrm{c}$ respectively. The LEV formation is detected at the leading edge where the pressure distribution is found to fluctuates. The LEV location at upper surface is indicated by the pressure peak on the lower line.

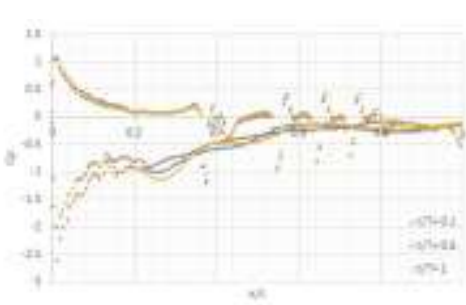

a.

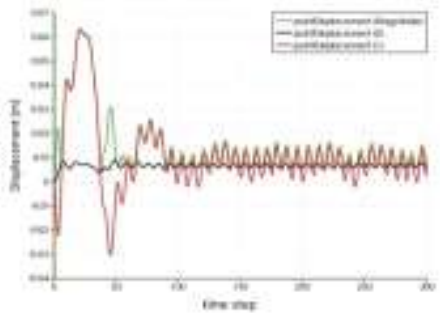

b.

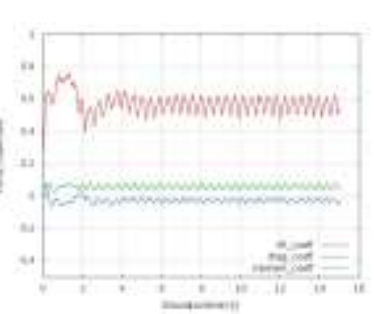

C.

Figure 5. a. Pressure distribution on the foil's surface, b. Displacement of the foil trailing edge, c. Force coefficient time history

A fluctuating pressure is denoted by an oscillation pressure distribution line in the direction of the flow (x direction). The separation which leads to vorticity is produced in the high pressure region. The region is recognized by 
a pressure peak in the pressure distribution profile. This agrees with Lee and Su's result (2015).

From Figure 5a, it is seen that the pressure distribution peak occurs at $\mathrm{x} / \mathrm{c}=0.261$. At the same location, the vortex formation is found which is shown in Figure 6 (pink node). Figure 6 illustrates the phenomenon of vortex and separation occurring in the location. The peak indicates vortex generation and separation. Figure 6 corresponds to Figure 4 and 5a. When $\mathrm{t} / \mathrm{T}=0.1$ the foil begins to move upward from its initial position and small vortex is detected to form at the upper surface at $x / c=0.216$ (Figure 6a). At the uppermost position (Figure $6 \mathrm{~b}$ ) the suction effect is reducing which cause the foil to move downward to lowest position and smoothen the vortex (Figure 6c). At the lowest position, the separation begins to occur starting from $\mathrm{x} / \mathrm{c}=0.246$ and move downward to trailing edge along with upward movement of foil.

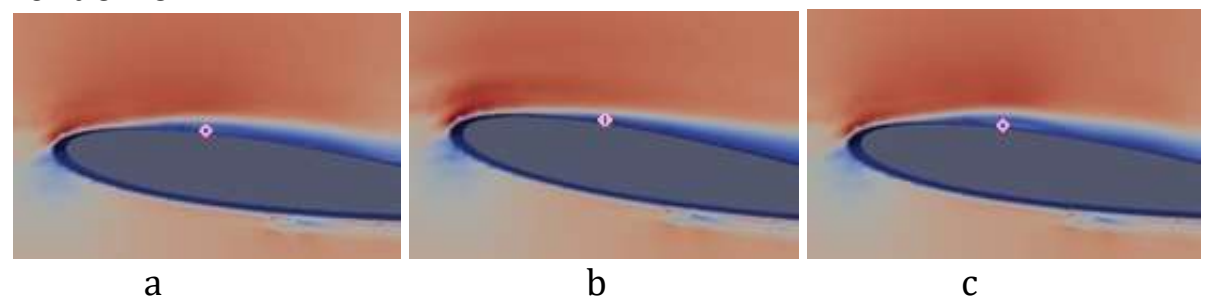

Figure 6. Vorticity on $\mathrm{x} / \mathrm{c}=0.216$ at $\mathrm{t} / \mathrm{T}=\mathrm{a} .0 .1, \mathrm{~b} .0 .6, \mathrm{c} .1$.

The fluctuating pressure which is generated over the foil surfaces induces fluctuating lift forces. The unsteady lift force causes foil to vibrate which can be indicated in the foil's displacement plot as depicted in Figure $5 b$. The vibration plot revelas that no high amplitude exists which indicates no lock-in condition. This result is in good agreement with the discussion from the dominant frequency plot in Figure 2c which shows no dominant signal approaching heave or pitch natural frequency.

- Displacement

The displacement which is taken at the foil's trailing edge is illustrated in Figure 5b. The lines indicate displacements in $x$ direction (pointDisplacement 0) and y direction (pointDisplacement 1) in 300 time steps. The 300 time steps represents fifteen seconds simulation time.

At the early timesteps until 60 (3 seconds simulation time), the vibration is found to be unsteady and irregular. This is consistent to velocity recording as shown in Figure 2c. From y direction motion which reflects heave vibration, the trailing edge vibrates regularly with a peak to peak amplitude of $0.013 \mathrm{~m}$. The vibration response of the foil due to interaction with incoming fluid, is found to be laminar and regular. This implies that the foil experiences a weak vibration during the interaction. The small amplitude displacement also confirms that the lock-in condition does not appear on the foil. 
- Force coeffcients analysis

Figure $5 \mathrm{c}$ shows the moment, lift, and drag coefficients of the foil. The lift coefficient forces the foil to vibrate as indicated in Figure $5 b$ and associated with the process detailed in Figure 4. Those fluid induced forces working on the foil are regularly oscillating which demonstrates the occurance of vortex shedding. They verified the vortex generation from the lift coefficient fluctuation which is close to fluctuations examined in Figure $5 c$. The regular force signals are generated by an oscillating velocity signal with a low amplitude during the operation. The periodic forces are results of the foil and fluid interaction which produces a weak vortex shedding. The interaction is also recognized from the the foil vibrations as seen from the foil response behaviour in Figure 4.

The drag and moment coefficient are also found fluctuating regularly but have lower magnitude than the lift coefficient. As such the heave motion appears stronger than pitch on the foil as seen in Figure 4. The transverse vibration occurs stronger than torsional vibration but none of them are in nor close to the lock-in frequency band as shown in Figure $3 \mathrm{~b}$ thus the forces coefficient results are consistent to the frequency domain results.

\section{Conclusion}

A procedure for predicting lock-in on a FSI-CFD model of NACA 0012 foil with $R e$ of $3.07 \times 10^{6}$ is proposed in this paper. The lock-in is identified from foil's vibration as the foil-fluid interaction response. The procedure employs two methods to identify the vortex shedding velocity and frequency. The methods are the Phase Averaged Method and the Power Spectral Density. The procedure can predict lock-in on the NACA 0012 foil quite well. The prediction is justified with the foil's surface pressure distribution, vortex shedding capture on the foil's surface, trailing edge displacement and time history of force coefficients. For the Re and selected foil's mechanical properties due to spring and damping characteristic, the foil does not show to be close to foil's natural frequency. Therefore lock-in is not detected. This can be verified from the foil's regular oscillations with no high frequency and amplitude vibration presence. The force coefficients are also recorded with the lift coefficient appears the most. The typical foil is beneficial to be applied for a device which driven by lift force such as in vertical axis tidal turbine.

\section{References}

[1] S. Mittal, S. et al., Lock-in in Vortex-Induced Vibration. Journal of Fluid Mechanics, Vol 794, 565-594, 2016.

[2] Bishop, Richard Evelyn Donohue, and A. Y. Hassan, The Lift and Drag Forces on A Circular Cylinder Oscillating in A Flowing Fluid. In Proceedings of the Royal Society of London A: Mathematical, Physical and Engineering Sciences, volume 277, pages 51-75. The Royal Society, 1964.

[3] Fanny M Besem, Joshua D. Kamrass, Jeffrey P. Thomas, Deman Tang, Robert E. Kielb, Vortex Induced Vibration and Frequency Lock-In of 
An Airfoil At High Angles of Attack. Journal of Fluids Engineering, Vol. 138, No. 1, 011204, 2016.

[4] D. Rana, S. Patel, A. K. Onkar, M. Manjuprasad, Time Domain Simulation of Airfoil Flutter Using Fluid Structure Coupling Through FEM Based CFD Solver, InSymposium of Apllied Aerodynamics and Design of Aerospace vehicle, SAROD201112009 Nov 16

[5] A. Ducoin, J. Astol, F. Deniset, J. F. Sigrist, An Experimental and Numerical Study of The Hydroelastic Behavior of An Hydrofoil in Transient Pitching Motion. In First International Symposium on Marine Propulsors, Trondheim, Norway. 2009

[6] A. Ducoin, Y. L. Young, Hydroelastic Response and Stability of A Hydrofoil In Viscous Flow. Journal of fluids and structures, Vol. 38, 4057,2013

[7] Deniz Tolga Akcabay, Eun Jung Chae, Yin Lu Young, Antoine Ducoin, Jacques Andre Astolfi, Cavity Induced Vibration of Flexible Hydrofoils. Journal of Fluids and Structures, Vol 49, 463-484, 2014a.

[8] Deniz Tolga Akcabay, Young, Yin Lu Young, Influence of Cavitation on The Hydroelastic Stability of Hydrofoils. Journal of Fluids and Structures, Vol 49, 170-185, 2014b.

[9] Nu Rhahida Arini, Stepen R. Turnock, Mingyi Tan, Two-Dimensional Fluid-Structure Interaction Analysis of A Vertical Axis Tidal Turbine Blade Using Periodic Inflow Equivalence Model. Journal of Engineering for the Maritime Environment, vol 232, No. 1, 2018.

[10] P. Davies, G. Germain, B. Gaurier, A. Boisseau, D. Perreux, Evaluation of The Durability of Composite Tidal Turbine Blades. Philosophical Transactions of the Royal Society of London A: Mathematical, Physical and Engineering Sciences, Vol 371, No. 20120187, 2013

[11] O. De La Torre, X. Escaler, E. Egusquiza, M. Farhat, Experimental Investigation Of Added Mass Effects on A Hydrofoil Under Cavitation Conditions. Journal of Fluids and Structures, Vol. 39, 73-187, 2013

[12] E Da Lozzo, F. Auricchio, G. M. Calvi, Added Mass Model for Vertical Circular Cylinder Partially Immersed in Water. Proceedings of 15th WCEE, Lisboa, 2012

[13] H. Ghassemi, E. Yari, The Added Mass Coefficient Computation of Sphere, Ellipsoid and Marine Propellers Using Boundary Element Method. Polish Maritime Research, Vol. 18, No 1, 17-26. 2011

[14] J. Klaassen, A Method of Manufacturing A Turbine Blade and A Tubine Blade. WO Patent App. PCT/EP2015/053,694, 2015

[15] R. Tabassian, Torsional Vibration Analysis of Shafts Based On Adomian Decomposition Method, Applied and Computational Mechanics, Vol 7, 205-222, 2013

[16] D.D Chung, Materials for Vibration Damping. Journal of materials science, Vol 36, No 24, 5733-5737, 2001 
[17] D. P. S. Kumar, K. Karthik. T. Raja, Vibration Damping Characteristics of Hybrid Polymer Matrix Composite. International Journal of Mechanical \& Mechatronics Engineering IJMME-IJENS, Vol. 15, No 01, 153101-8282, 2015

[18] A. Hussain, W. Reynolds, The Mechanics of An Organized Wave In Turbulent Shear Flow. Journal of Fluid Mechanics, Vol 41, No 2, 241258,1970

[19] A. Hussain, W. Reynolds, The Mechanics of An Organized Wave in Turbulent Shear Flow. Part 2. Experimental Results. Journal of Fluid Mechanics, Vol. 54, No 02, 241-261, 1972

[20] Y. Jung, S. Park, Vortex-Shedding Characteristics in The Wake of An Oscillating Airfoil At Low Reynolds Number. Journal of Fluids and Structures, Vol 20, No 3, 451-464. 2005

[21] F. Ostermann, R. Woszidlo, S. Gaertlein, C. Nayeri, C. O. Paschereit, Phase-Averaging Methods For A Naturally Oscillating Flow Flield, In 52nd Aerospace Sciences Meeting, page 1142, 2014

[22] JA Bourgeois, BR Noack, RJ Martinuzzi, Generalized Phase Average with Applications to Sensor-Based Flow Estimation of The WallMounted Square Cylinder Wake. Journal of Fluid Mechanics, Vol. 736, 316-350, 2013a.

[23] JA Bourgeois, BR Noack, RJ Martinuzzi, Generalized Phase Averaging of Experimental Surface-Mounted Body Wake Measurements: 3D Coherent Structures \& Dynamical Models. In TSFP DIGITAL LIBRARY ONLINE. Begel House Inc. 2013b.

[24] R. Perrin, M. Braza, E. Cid, S. Cazin, A. Barthet, A. Sevrain, C. Mockett, F. Thiele, Obtaining Phase Averaged Turbulence Properties in The Near Wake of A Circular Cylinder At High Reynolds Number Using POD. In Proc. 13th Int. Symp. Applications of Laser Techniques to Fluid Mechanics, Lisbon, Portugal. 2007

[25] R. Perrin, M. Braza, E. Cid, S. Cazin, F. Moradei, A. Barthet, A. Sevrain, Y. Hoarau, Near-Wake Turbulence Properties in The High Reynolds Number Incompressible Flow Around A Circular Cylinder Measured by Two-And Three-Component PIV. Flow, Turbulence and Combustion, Vol 77, No. 1, 185-204, 2006

[26] Robert. D Blevins, Flow-induced vibration. Van Nostrand Reinhold Co. Inc, New York, NY (USA), 1990

[27] T. Lee, Y. Su, Y, Surface Pressures Developed on An Airfoil Undergoing Heaving and Pitching Motion. Journal of Fluids Engineering, Vol 137, No. 5, 051105, 2015 